\title{
High-dose Sulbactam Treatment for Ventilator-Associated Pneumonia Caused by Carbapenem-Resistant Acinetobacter Baumannii
}

In Beom Jeong, M.D., Moon Jun Na, M.D., Ji Woong Son, M.D., Do Yeon Jo, M.D., and Sun Jung Kwon, M.D.

Department of Internal Medicine, Konyang University College of Medicine, Daejeon, Korea

Background: Several antibiotics can be used to treat ventilator-associated pneumonia caused by carbapenem-resistant $A$. baumannii (CRAB-VAP) including high-dose sulbactam. However, the effectiveness of high-dose sulbactam therapy is not well known. We report our experience with high-dose sulbactam for treatment of CRAB-VAP.

Methods: Medical records of patients with CRAB-VAP who were given high-dose sulbactam between May 2013 and June 2015 were reviewed.

Results: Fifty-eight patients with CRAB-VAP were treated with high-dose sulbactam. The mean age was $72.0 \pm 15.2$ years, and the acute physiology and chronic health evaluation II (APACHE II) score was $15.1 \pm 5.10$ at the time of CRAB-VAP diagnosis. Early clinical improvement was observed in $65.5 \%$ of patients, and 30 -day mortality was $29.3 \%$. Early clinical failure (odds ratio [OR]: 8.720 , confidence interval [CI]: 1.346-56.484; $p=0.023$ ) and APACHE II score $\geq 14$ at CRAB-VAP diagnosis (OR: 10.934, Cl: 1.047-114.148; $p=0.046$ ) were associated with 30-day mortality.

Conclusions: High-dose sulbactam therapy may be effective for the treatment of CRAB-VAP. However, early clinical failure was observed in $35 \%$ of patients and was associated with poor outcome.

Key Words: acinetobacter; pneumonia, ventilator-associated; sulbactam.

\section{Introduction}

Acinetobacter baumannii (A. baumannii) a non-lactose-fermenting, gram-negative coccobacillus found extensively in nature, has emerged as a major cause of nosocomial infection in critical care settings.[1] This microorganism is characterized by the rapid acquisition of resistance to multiple antibiotics[2] and survives longer in inanimate objects such as ventilator circuits, rubber, steel, and plastic and can colonize anywhere in the intensive care unit (ICU).[3]

Due to its ability to survive for an extended period of time on the surfaces of medical equipment and to acquire multiple antibiotic resistance, A. baumannii is a common cause of nosocomial infection, [4] causing a wide range of infec-

Received on December 17, 2015 Revised on August 12, 2016 Accepted on August 18, 2016

Correspondence to: Sun Jung Kwon, Department of Internal Medicine, Konyang University College of Medicine, 158 Kwanjodong-ro, Seo-gu, Daejeon 35365, Korea

Tel: +82-42-600-9100, Fax: +82-42-600-9090

E-mail: sjoongkwon@hanmail.net

*No potential conflict of interest relevant to this article was reported. tions including wound infections, urinary tract infections, meningitis, bacteremia, and ventilator-associated pneumonia (VAP). In VAP, carbapenem-resistant A. baumannii (CRAB) is currently one of the most problematic pathogens because the antimicrobial choices are limited due to the inherent and acquired resistance of the microorganism.

Several trials have evaluated effective antimicrobial 
agents for CRAB infections. The recommended antibiotic options are limited but include minocycline, tigecycline, and polymyxins. Minocycline and tigecycline have broad-spectrum in vitro activity against several multidrug-resistant strains of $A$. baumannii, suggesting them as suitable candidates for serious infections. However, data supporting clinical use, particularly in VAP caused by CRAB (CRAB-VAP), are limited.[5-7] Colistin, belonging to the polymyxin group, was first discovered in 1949 and became available in 1959.[8] However, due to the high incidence of adverse effects, colistin was discontinued for an extended period of time. Although multidrugresistant organisms such as $\mathrm{CRAB}$ compelled clinicians to reintroduce colistin to clinical practice recently, the adverse effects and development of resistance remain major concerns. Several recent studies showed that the incidence of colistin-associated nephrotoxicity has increased to approximately $40-43 \%$. The use of aerosolized colistin to reduce nephrotoxicity remains controversial, $[9,10]$ and clinicians use it with a high burden of toxicity.

Sulbactam is an irreversible inhibitor of beta-lactamase and is typically administered in combination with betalactam antibiotics. Although most beta-lactamase inhibitors do not exert antimicrobial activities, sulbactam has shown to have in vitro activity against Acinetobacter species.[11-13] Previous data from observational studies suggest that ampicillin-sulbactam is comparable in efficacy to imipenem in patients with $A$. baumannii bacteremia.[14] Strains resistant to carbapenems show in vitro susceptibility to sulbactam;[15] however, limited data exists supporting the therapeutic effects of sulbactam in CRAB-VAP. This study was designed to estimate the therapeutic effects of high-dose sulbactam and factors associated with poor outcome.

\section{Materials and Methods}

\section{1) Study design}

This was a retrospective case series study conducted in the 58-bed adult ICU of a university hospital in Daejeon,
Korea. The study was approved by the Institutional Review Board.

Medical records of patients who received ampicillinsulbactam or piperacillin-sulbactam for CRAB-VAP between May 2013 and June 2015 were reviewed. Patients given high-dose sulbactam for CRAB-VAP treatment were enrolled in the study. Patients given any CRABtargeted antimicrobial therapy before sulbactam were excluded, as were patients for whom sulbactam was delayed more than 24 hours after confirmation of CRABVAP diagnosis. The collected data included demographic characteristics, comorbidities, severity of disease, use of invasive procedures, laboratory results, antimicrobial therapy, and clinical outcome. The severity of illness was assessed using the Acute Physiology and Chronic Health Evaluation II (APACHE II) score on the first day of ICU admission and on the day of CRAB-VAP diagnosis.[16] Severity was also assessed using the modified Clinical Pulmonary Infection Score (CPIS) on the day of CRAB-VAP diagnosis and again five days later.[17] The Sequential Organ Failure Assessment (SOFA) score was calculated daily to monitor changes in severity.[18]

\section{2) Definitions}

Diagnosis of pneumonia required the presence of new or persistent pulmonary infiltrates. Additionally, at least two of the following criteria were required: (1) body temperature $>38.4^{\circ} \mathrm{C}$ or $<36.0^{\circ} \mathrm{C}$, (2) leukocyte count $>11,000 / \mathrm{mm}^{3}$ or $<4000 / \mathrm{mm}^{3}$, and (3) purulent sputum. Pneumonia that developed after 72 hours of mechanical ventilation was considered VAP.

The etiology of VAP was established by isolation of the organism from endotracheal aspirates or bronchoalveolar lavage with moderate or heavy growth based on semi-quantitative culture, or concentration $\geq 10^{4} \mathrm{CFU} /$ $\mathrm{mL}$ using a quantitative technique. Because the isolates of $A$. baumannii are often multidrug resistant,[19] CRAB was defined as $A$. baumannii resistant to multiple antibiotics including meropenem and/or imipenem-cilastatin, excluding colistin and tigecycline. CRAB-VAP was diagnosed after confirmation of CRAB in VAP patients. 
Clinical outcome was divided as early clinical improvement or early clinical failure. Early clinical improvement was assessed using five CPIS items (fever, leukocyte count, oxygenation status, quantity and purulence of tracheal secretions, and radiographic abnormality) and defined as decreased modified CPIS after five days of sulbactam treatment. A score greater than or equal to the previous CPIS was considered an early clinical failure.

Microbiological outcome was classified as microbiological improvement or microbiological failure. Microbiological improvement was defined as the eradication of pathogen (no pathogen growth in two consecutive cultures after treatment), and microbiological failure was defined as persistent $A$. baumannii in any sputum culture after treatment.

Decrease of kidney function was defined as a decrease of estimated glomerular filtration rate (eGFR) $>20 \mathrm{~mL} / \mathrm{min} /$ $1.73 \mathrm{~m}^{2}$ during five days of treatment and was calculated using the Chronic Kidney Disease Epidemiology Collaboration formula.[20] SOFA score increase was defined as an increase in the score during five days of treatment.

\section{3) Drug administration}

For most patients, sulbactam was administered at a daily dose of $8 \mathrm{~g}$. Approximately eight vials of piperacillin-sulbactam (16 $\mathrm{g}$ piperacillin, $8 \mathrm{~g}$ sulbactam) were administered, and 32 vials of ampicillin-sulbactam (16 $\mathrm{g}$ ampicillin, $8 \mathrm{~g}$ sulbactam) were administered per day. The attending physician adjusted the dose based on the patient's body weight and kidney function. In our hospital, cefoperazone-sulbactam was not available and thus was not included in this study.

\section{4) Microbiological methods}

Sputum samples were collected by bronchoscopic lavage or endotracheal suction. Microbial identification and susceptibility testing were conducted using the MicroScan WalkAway Plus System (Siemens, Deerfield, IL, USA). Antimicrobial susceptibility was determined using the minimal inhibitory concentration (MIC) of antibiotics according to the Clinical and Laboratory Standards Insti- tute guidelines.[21] Tested antibiotics were aminoglycoside, ampicillin-sulbactam, cefepime, cefotaxime, piperacillin, piperacillin-tazobactam, meropenem, tigecycline, and colistin.

\section{5) Statistical analysis}

Continuous variables were compared using the MannWhitney $U$ test or paired $t$-test when appropriate. Categorical variables were compared using Pearson's Chi square test or Fisher's exact test. Variables from univariate analyses with a p-value $<0.05$ were candidates for multivariate analysis. Univariate and multivariate analyses of risk factors associated with 30-day mortality were performed using Cox proportional hazards regression models. The statistical computing program R-3.1.3 was used for the statistical analyses.

\section{Results}

During the study period, 58 CRAB-VAP patients were treated with high-dose sulbactam. Table 1 shows the demographic and clinical characteristics of the patients. Most patients were male $(38 ; 66 \%)$, and the mean age was $72 \pm 15.2$ years. The mean Charlson comorbidities index was $6.4 \pm 2.4$, and chronic obstructive pulmonary disease was the most common underlying respiratory disease (n $=27 ; 46.6 \%$ ). The mean hospital stay before CRAB-VAP was $19.4 \pm 22.4$ days, and the mean ICU stay was $15.5 \pm$ 17.8 days. The mean APACHE II score was $20.5 \pm 15.2$ at ICU admission and $15.1 \pm 5.10$ at CRAB-VAP diagnosis. At the day of CRAB-VAP diagnosis, CPIS was $6.25 \pm 1.5$, eGFR was $81.7 \pm 42.2 \mathrm{~mL} / \mathrm{min} / 1.73 \mathrm{~m}^{2}$, and SOFA was 5.3 \pm 3.2. All CRAB showed resistance to multiple antibiotics including ampicillin-sulbactam (MIC $>16 / 8 \mu / \mathrm{mL}$ ), meropenem (MIC $>8 \mu / \mathrm{mL}$ ), and imipenem ( $\mathrm{MIC}>8 \mu / \mathrm{mL}$ ), but did not show resistance to colistin (MIC $\leq 2 \mu / \mathrm{mL}$ ) or tigecycline (MIC $\leq 2 \mu / \mathrm{mL}$ ).

A daily dose of sulbactam ( $8 \mathrm{~g} /$ day $)$ was given to most patients. Because the dose was adjusted to the patient's eGRF and body weight, the average sulbactam dose for 
Table 1. Demographics and clinical characteristics of patients treated with high-dose sulbactam

\begin{tabular}{lc}
\hline & Patients $(\mathrm{n}=58)$ \\
\hline Mean age (years) & $72.0 \pm 15.2$ \\
\hline Male gender & $38(65.5)$ \\
\hline BMI & $20.6(3.2)$ \\
\hline ABW (kg) & $53.7(11.4)$ \\
\hline Charlson comorbidities index & $6.4 \pm 2.4$ \\
\hline Underlying disease & \\
\hline Diabetes mellitus & $12(20.6)$ \\
\hline Hypertension & $31(53.4)$ \\
\hline Chronic obstructive pulmonary disease & $27(46.6)$ \\
\hline Chronic kidney disease & $11(18.8)$ \\
\hline Congestive heart failure & $18(31.0)$ \\
\hline Cerebrovascular accident & $25(43.1)$ \\
\hline Malignancy & $7(12.1)$ \\
\hline Length of hospital stay, days (IQR) & $19.4 \pm 22.4$ \\
\hline Length of ICU stay, days (IQR) & $15.5 \pm 17.8$ \\
\hline APACHE II at ICU admission (IQR) & $20.5 \pm 15.2$ \\
\hline APACHE $\|$ at CRAB-VAP diagnosis (IQR) & $15.1 \pm 5.10$ \\
\hline CPIS at CRAB-VAP diagnosis & $6.25 \pm 1.5$ \\
\hline eGFR at CRAB-VAP diagnosis & $81.7 \pm 42.2$ \\
\hline SOFA at CRAB-VAP diagnosis & $5.3 \pm 3.2$ \\
\hline Co-infection with other organism & $23(39.6)$ \\
\hline MRSA & $20(34.5)$ \\
\hline Pseudomonas & $2(3.4)$ \\
\hline
\end{tabular}

Values are presented as mean \pm SD or $n(\%)$.

BMl: body mass index; ABW: actual body weight; IQR: interquartile range; ICU: intensive care unit; APACHE II: acute physiology and chronic health evaluation II; CRAB: carbapenem-resistant Acinetobacter baumannil; VAP: ventilator-associated pneumonia; CPIS: clinical pulmonary infection score; eGFR: estimated glomerular filtration rate; SOFA: sequential organ failure assessment; MRSA: Methicillin-resistant Staphylococcus aureus.

all patients was $7 \mathrm{~g} /$ day. The mean body weight was 53.7 $\pm 11.4 \mathrm{~kg}$, and $130 \pm 64 \mathrm{mg} / \mathrm{kg}$ of sulbactam was administered. Twenty-six patients received ampicillin-sulbactam and 32 received piperacillin sulbactam. Sulbactam dose was not significantly different between the two sulbactam-containing antibiotics $(132 \pm 73 \mathrm{mg} / \mathrm{kg}$ for ampicillin-sulbactam vs. $129 \pm 51 \mathrm{mg} / \mathrm{kg}$ for piperacillinsulbactam, $\mathrm{p}=0.850$ ).

Among the patients, 17 (29.3\%) died within 30 days after CRAB-VAP diagnosis; 15 -day mortality was $12.1 \%$. Table 2 shows the univariate analysis of potential risk factors for 30-day mortality. Among the variables, age $(\mathrm{p}=0.002)$, APACHE II score $>14$ at CRAB-VAP diagnosis $(p=0.002)$, eGRF decrease $(p=0.001)$, SOFA increase $(p=0.015)$, and early clinical failure $(p=0.000)$, were associated with 30-day mortality. There were no significant differences regarding the type of combined beta-lactam (ampicillin $69.2 \%$ vs. $30.8 \%$; piperacillin $56.1 \%$ vs. $52.9 \%, \mathrm{p}=1.000$ ), gender (male $70.7 \%$ vs. $52.9 \%, \mathrm{p}=0.502)$, APACHE II score at ICU admission (20.1 vs. $22.0, \mathrm{p}=0.286$ ), days of hospital stay (22.9 vs. $14.9, \mathrm{p}=0.216)$, or ICU stay before CRAB-VAP (18.2 days vs. 12.4 days, $\mathrm{p}=0.192$ ). Charlson comorbidities index, eGFR, SOFA, and CPIS at CRAB-VAP diagnosis were also not associated with mortality. Several microorganisms were isolated together with CRAB. Methicillinresistant Staphylococcus aureus (MRSA) was identified in 20 patients (34.5\%) and Pseudomonas in 2 patients (3.4\%). Due to the high rate of co-infection with MRSA, glycopeptide antibiotics were the most commonly administered with sulbactam $(\mathrm{n}=26,44.8 \%)$. Several other antibiotics were also given simultaneously $(\mathrm{n}=35$, $60.3 \%$ ); however, no significant difference in mortality was observed regarding co-administered antibiotics or co-infection with other microorganisms.

Multivariate analysis using a logistic regression model showed that the factors associated with 30-day mortality were early clinical failure (odds ratio [OR]: 8.720, confidence interval [CI]: 1.35-56.48; $\mathrm{p}=0.023$ ) and APACHE II score $\geq 14$ at CRAB-VAP diagnosis (OR: 10.934, CI: 1.05-114.15; $\mathrm{p}=0.046$; Table 3).

\section{Discussion}

Several previous studies evaluated the efficacy of high-dose sulbactam for the treatment of CRAB-VAP. However, because the studies were conducted with small populations, mortality predictors were not assessed. Betrosian et al.[22] conducted a prospective study comparing the efficacy of ampicillin-sulbactam and colistin for the treatment of CARB-VAP. In their study, 13 patients 
Table 2. Clinical and demographic characteristic differences between survivors and non-survivors

\begin{tabular}{|c|c|c|c|}
\hline & Survivor $(n=41)$ & Non-survivor $(n=17)$ & $p$-value \\
\hline Ampicillin-sulbactam & $18(69.2)$ & $8(30.8)$ & 0.826 \\
\hline Piperacillin-sulbactam & $23(56.1)$ & $9(52.9)$ & \\
\hline Sulbactam dose (mg/kg) & $122 \pm 51$ & $151 \pm 87$ & 0.208 \\
\hline Male gender & $29(70.7)$ & $9(52.9)$ & 0.502 \\
\hline Age (years) & $69.5 \pm 14.3$ & $77.8 \pm 16.1$ & 0.002 \\
\hline APACHE $\|$ at ICU admission & $19.7 \pm 7.7$ & $22.3 \pm 7.1$ & 0.188 \\
\hline APACHE $\|$ at CRAB-VAP diagnosis & $13.5 \pm 4.4$ & $19.0 \pm 4.5$ & 0.001 \\
\hline APACHE $\| \geq 14$ at CRAB-VAP diagnosis & $21(51.2)$ & $16(94.1)$ & 0.002 \\
\hline Length of hospital stay, days & $22.2 \pm 25.8$ & $12.7 \pm 7.8$ & 0.216 \\
\hline Length of ICU stay, days & $17.5 \pm 20.5$ & $10.8 \pm 7.5$ & 0.192 \\
\hline Charlson comorbidities index & 6.19 & 7.00 & 0.139 \\
\hline Diabetes mellitus & $8(19.5)$ & $4(23.5)$ & 0.733 \\
\hline COPD & $20(48.8)$ & $7(41.2)$ & 0.773 \\
\hline Hypertension & $22(53.7)$ & $9(52.9)$ & 0.960 \\
\hline Cerebrovascular accident & $16(39.0)$ & $9(52.9)$ & 0.390 \\
\hline Chronic kidney disease & $8(19.5)$ & $3(17.6)$ & 0.869 \\
\hline Congestive heart failure & $12(29.3)$ & $6(35.3)$ & 0.758 \\
\hline Malignancy & $6(14.6)$ & $1(5.9)$ & 0.329 \\
\hline Co-infection with other organism & $18(43.9)$ & $5(29.4)$ & 0.304 \\
\hline MRSA & $17(41.5)$ & $3(17.6)$ & 0.082 \\
\hline Pseudomonas & $1(2.4)$ & $1(5.9)$ & 0.513 \\
\hline Others & $1(2.4)$ & $1(5.9)$ & 0.504 \\
\hline \multicolumn{4}{|l|}{ Combined antibiotics } \\
\hline Carbapenem & $4(9.8)$ & $1(5.9)$ & 1.000 \\
\hline Glycopeptide & $18(43.9)$ & $8(47.1)$ & 0.826 \\
\hline Quinolone & $5(12.2)$ & $2(11.8)$ & 1.000 \\
\hline Aminoglycoside & $7(17.1)$ & $4(23.5)$ & 0.715 \\
\hline Cephalosporin & $2(4.9)$ & $2(11.8)$ & 0.573 \\
\hline Antipseudomonal beta-lactam & $3(7.3)$ & $1(5.9)$ & 1.000 \\
\hline Others & $1(2.4)$ & $3(17.6)$ & 0.071 \\
\hline CPIS at CRAB-VAP diagnosis & $6.45 \pm 1.6$ & $5.76 \pm 1.1$ & 0.061 \\
\hline Early treatment failure & $7(17.1)$ & $13(76.5)$ & $<0.001$ \\
\hline SOFA at CRAB-VAP diagnosis & $5.1 \pm 3.4$ & $5.9 \pm 2.5$ & 0.143 \\
\hline SOFA score increase & $12(30.0)$ & $11(64.7)$ & 0.020 \\
\hline Microbiologic improvement & $27(65.9)$ & $5(38.5)$ & 0.109 \\
\hline Radiologic improvement & $25(61.0)$ & $7(41.2)$ & 0.247 \\
\hline
\end{tabular}

APACHE II: acute physiology and chronic health evaluation I; ICU: intensive care unit; COPD: chronic obstructive pulmonary disease; MRSA: Methicillin-resistant Staphylococcus aureus; CPIS: clinical pulmonary infection score; CRAB: carbapenem-resistant Acinetobacter baumanniï; VAP: ventilator-associated pneumonia; SOFA: sequential organ failure assessment.

were treated with high-dose ampicillin-sulbactam $(9 \mathrm{~g} /$ day as sulbactam), and the mean APACHE II score was $14 \pm 5$. Clinical improvement was observed in $69.1 \%$ of the patients, $15.3 \%$ died within 14 days, and 28-day mortality was $30.0 \%$. The clinical outcome of high-dose sulbactam was not significantly worse than that of co- 
Table 3. Risk factors of 30-day mortality in carbapenem-resistant Acinetobacter baumannii ventilator-associated pneumonia

\begin{tabular}{lrcc}
\hline & Odds ratio & Confidence Intervals & $p$-value \\
\hline Early clinical failure & 8.720 & $1.35-56.48$ & 0.023 \\
APACHE $\| \geq 14$ & 10.934 & $1.05-114.15$ & 0.046 \\
\hline
\end{tabular}

APACHE II: acute physiology and chronic health evaluation II.

listin (28-day mortality: 33.3\%). In another study by the same authors, a comparison between two different doses of sulbactam (9 g/day vs. $12 \mathrm{~g}$ /day) was conducted.[23] Clinical improvement was observed in $66.7 \%$ of the study population, and the 30-day mortality was $48.1 \%$; no significant difference in mortality rate was observed between the groups. Wood et al.[24] retrospectively studied 77 CRAB-VAP patients; 14 patients were treated with ampicillin-sulbactam, and 63 patients were treated with imipenem-cilastatin. However, the total dose of sulbactam administered was not provided. In the ampicillinsulbactam group, clinical improvement was observed in $93 \%$ of patients, and there were no mortalities (17\% mortality in the imipenem group). In another study, 12 patients were treated with ampicillin-sulbactam, 75\% of whom showed clinical improvement.[25] In the present study, clinical improvement was observed in $65.5 \%$ of patients, and the 30-day mortality rate was $29.3 \%$. Clinical outcome was consistent with previous studies that used sulbactam as a therapeutic agent. A majority of previous studies evaluating the efficacy of colistin-based therapy for CRAB-VAP reported a 30-day mortality of 27-43\%.[26-29] Based on these results, possible noninferiority of high-dose sulbactam to colistin in CRABVAP treatment can be assumed.

Factors such as APACHE II score at CRAB-VAP diagnosis, early clinical failure, SOFA score increase, and increasing age were associated with higher 30-day mortality in univariate analyses. Worsening of pneumonia that leads to death is usually accompanied by multi-organ failure. Hence, the association between SOFA score increase and mortality is reasonable. Increasing age also showed significant association with 30-day mortality. To adjust covariates, multivariate analyses were performed. The risk factors of 30-day mortality in patients with
CRAB-VAP treated with high-dose sulbactam were early clinical failure and APACHE II score at CRAB-VAP diagnosis. APACHE II score is a widely used prognosis evaluation tool for ICU patients. However, in the present study, no association was found between APACHE II score at ICU admission and 30-day mortality. Due to long ICU stays (15.5 days) before CRAB-VAP diagnosis, APACHE II scores at the first day of ICU admission did not correlate with the mortality of patients later diagnosed with CRAB-VAP. To avoid selection bias, we also calculated the APACHE II score at CRAB-VAP diagnosis. There was a strong correlation between 30-day mortality and APACHE II score at CRAB-VAP diagnosis; APACHE II score $>14$ showed the strongest association. We believe recalculating APACHE score when VAP develops is useful for estimating the prognosis.

Early clinical failure, which was determined five days after high-dose sulbactam treatment, was strongly associated with 30-day mortality. Since early clinical failure is a risk factor of mortality, we recommend considering a prompt change of antibiotic in early clinical failure. Although antibiotic options for CRAB-VAP are limited, we consider an antibiotic change more reasonable than waiting for improvement with successive sulbactam administration.[30]

We administered approximately $8 \mathrm{~g}$ of sulbactam daily to most patients. Dose was modified by the attending physician according to the patient's body weight and renal function. Mean administered dosage was $7 \mathrm{~g}$. A previous study showed no difference in clinical outcomes between $9 \mathrm{~g}$ /day and $12 \mathrm{~g} /$ day dosages.[23] Another study, in which $4 \mathrm{~g}$ of sulbactam was administered per day, $60.0 \%$ of patients showed clinical improvement. However, the study results did not show the efficacy of relatively low-dose sulbactam treatment because sulbactam was administered with colistin in some patients, and the number of VAP patients was too small.[31] We suggest that a daily sulbactam dose of $8 \mathrm{~g}$ is reasonable for patients with average weight and favorable renal function.

In this study, two forms of sulbactam were used, piper- 
acillin-sulbactam and ampicillin-sulbactam. This is the first study that used piperacillin-sulbactam as a sulbactam regimen for treatment of CRAB-VAP. No differences were observed between the two regimens with regard to clinical outcomes of mortality, early treatment failure, and decrease in renal function. Although a small number of patients were used for analysis, piperacillin-sulbactam showed equal therapeutic efficacy to ampicillin-sulbactam.

Among the CRAB-VAP patients, co-infection with other microorganisms was observed in $39.6 \%$ of patients; MRSA was the most common microorganism. Among $20(34.5 \%)$ patients infected with MRSA concurrently with CRAB, 17 (41.5\%) were in the survivor group, and three $(17.6 \%)$ were in the mortality group. Eighteen (43.9\%) patients in the survivor group and eight (47.1\%) patients in the mortality group were given a glycopeptide antibiotic and sulbactam; however, no significant difference was observed for the glycopeptide combination for MRSA co-infection ( $p=0.826$ and $p=0.082$, respectively). Because the glycopeptide has no antibacterial activity against CRAB, we hypothesized that it does not affect CRAB-VAP treatment outcome. Several other types of antibiotics were co-administered in $79.3 \%$ patients; none of these antibiotics had an antibacterial effect on CRAB. There was no difference in 30-day mortality regardless of antibiotic combination (sulbactam monotherapy group: $25 \%$ vs. combination group: $30.4 \% ; \mathrm{p}=0.713$ ).

This study had several limitations. The study was adapted from a single center, and a small number of patients were enrolled. Additionally, the study was conducted retrospectively, so selection bias may have occurred. Since treatment was administered by several attending physicians from the medical or surgical ICU, the decisions were physician-dependent despite similar clinical situations. Radiologic review was performed by only one physician; thus, the validity of the review cannot be guaranteed.

We conclude that high-dose sulbactam can be effective for the treatment of CRAB-VAP. However, early clinical failure is common and is associated with higher mortality with this treatment. Therefore, we recommend prompt change of antibiotics when clinical deterioration is observed during the early stages of high-dose sulbactam treatment. A larger prospective randomized controlled study is recommended.

ORCID

In Beom Jeong http://orcid.org/0000-0001-9587-5122

\section{References}

1) Dijkshoorn L, Nemec A, Seifert H: An increasing threat in hospitals: multidrug-resistant Acinetobacter baumannii. Nat Rev Microbiol 2007; 5: 939-51.

2) Lortholary O, Fagon JY, Hoy AB, Slama MA, Pierre J, Giral P, et al: Nosocomial acquisition of multiresistant Acinetobacter baumannii: risk factors and prognosis. Clin Infect Dis 1995; 20: 790-6.

3) Jawad A, Heritage J, Snelling AM, Gascoyne-Binzi DM, Hawkey PM: Influence of relative humidity and suspending menstrua on survival of Acinetobacter spp. on dry surfaces. J Clin Microbiol 1996; 34 : 2881-7.

4) Fournier PE, Richet H: The epidemiology and control of Acinetobacter baumannii in health care facilities. Clin Infect Dis 2006; 42: 692-9.

5) Castanheira M, Mendes RE, Jones RN: Update on Acinetobacter species: mechanisms of antimicrobial resistance and contemporary in vitro activity of minocycline and other treatment options. Clin Infect Dis 2014; 59(Suppl 6): S367-73.

6) Halstead DC, Abid J, Dowzicky MJ: Antimicrobial susceptibility among Acinetobacter calcoaceticusbaumannii complex and Enterobacteriaceae collected as part of the Tigecycline Evaluation and Surveillance Trial. J Infect 2007; 55: 49-57.

7) Karageorgopoulos DE, Kelesidis T, Kelesidis I, Falagas ME: Tigecycline for the treatment of multidrugresistant (including carbapenem-resistant) Acineto- 
bacter infections: a review of the scientific evidence. J Antimicrob Chemother 2008; 62: 45-55.

8) Kumazawa J, Yagisawa M J: The history of antibiotics: the Japanese story. Infect Chemother 2002; 8: 125-33.

9) Pogue JM, Lee J, Marchaim D, Yee V, Zhao JJ, Chopra $\mathrm{T}$, et al: Incidence of and risk factors for colistinassociated nephrotoxicity in a large academic health system. Clin Infect Dis 2011; 53: 879-84.

10) Rocco M, Montini L, Alessandri E, Venditti M, Laderchi A, De Pascale G, et al: Risk factors for acute kidney injury in critically ill patients receiving high intravenous doses of colistin methanesulfonate and/or other nephrotoxic antibiotics: a retrospective cohort study. Crit Care 2013; 17: R174.

11) Traub WH, Spohr M: Antimicrobial drug susceptibility of clinical isolates of Acinetobacter species (A. baumannii, A. haemolyticus, genospecies 3, and genospecies 6). Antimicrob Agents Chemother 1989; 33: 1617-9.

12) Vila J, Marcos A, Marco F, Abdalla S, Vergara $Y$, Reig R, et al: In vitro antimicrobial production of beta-lactamases, aminoglycoside-modifying enzymes, and chloramphenicol acetyltransferase by and susceptibility of clinical isolates of Acinetobacter baumannii. Antimicrob Agents Chemother 1993; 37: 138-41.

13) Fishbain J, Peleg AY: Treatment of Acinetobacter infections. Clin Infect Dis 2010; 51: 79-84.

14) Jellison TK, Mckinnon PS, Rybak MJ: Epidemiology, resistance, and outcomes of Acinetobacter baumannii bacteremia treated with imipenem-cilastatin or ampicillin-sulbactam. Pharmacotherapy 2001; 21: $142-8$.

15) Henwood CJ, Gatward T, Warner M, James D, Stockdale MW, Spence RP, et al: Antibiotic resistance among clinical isolates of Acinetobacter in the $\mathrm{UK}$, and in vitro evaluation of tigecycline (GAR936). J Antimicrob Chemother 2002; 49: 479-87.

16) Knaus WA, Draper EA, Wagner DP, Zimmerman JE: APACHE II: a severity of disease classification sys- tem. Crit Care Med 1985; 13: 818-29.

17) Pugin J, Auckenthaler R, Mili N, Janssens JP, Lew PD, Suter PM: Diagnosis of ventilator-associated pneumonia by bacteriologic analysis of bronchoscopic and nonbronchoscopic "blind" bronchoalveolar lavage fluid. Am Rev Respir Dis 1991; 143(5 Pt 1): 1121-9.

18) Vincent JL, Moreno R, Takala J, Willatts S, De Mendonça A, Bruining H, et al: The SOFA (Sepsis-related Organ Failure Assessment) score to describe organ dysfunction/failure. On behalf of the working group on sepsis-related problems of the European society of intensive care medicine. Intensive Care Med 1996; 22: 707-10.

19) Bou G, Otero FM, Santiso R, Tamayo M, Fernández Mdel C, Tomás M, et al: Fast assessment of resistance to carbapenems and ciprofloxacin of clinical strains of Acinetobacter baumannii. J Clin Microbiol 2012; 50: 3609-13.

20) Levey AS, Stevens LA, Schmid CH, Zhang YL, Castro AF 3rd, Feldman HI, et al: A new equation to estimate glomerular filtration rate. Ann Intern Med 2009; 150: 604-12.

21) Franklin R, Cockerill III: Performance standards for antimicrobial susceptibility testing; twenty-first informational supplement M100-S21. Wayne, Clinical and Laboratory Standards Institute; 2011.

22) Betrosian AP, Frantzeskaki F, Xanthaki A, Douzinas EE: Efficacy and safety of high-dose ampicillin/ sulbactam vs. colistin as monotherapy for the treatment of multidrug resistant Acinetobacter baumannii ventilator-associated pneumonia. J Infect 2008; 56: 432-6.

23) Betrosian AP, Frantzeskaki F, Xanthaki A, Georgiadis G: High-dose ampicillin sulbactam as an alternative treatment of late-onset VAP from multidrugresistant Acinetobacter baumannii. Scand J Infect Dis 2007; 39: 38-43.

24) Wood GC, Hanes SD, Croce MA, Fabian TC, Boucher BA: Comparison of ampicillin-sulbactam and imipenem-cilastatin for the treatment of acineto- 
bacter ventilator-associated pneumonia. Clin Infect Dis 2002; 34: 1425-30.

25) Levin AS, Levy CE, Manrique AE, Medeiros EA, Costa SF: Severe nosocomial infections with imipenem-resistant Acinetobacter baumannii treated with ampicillin/sulbactam. Int J Antimicrob Agents 2003; 21: 58-62.

26) Kim WY, Moon JY, Huh JW, Choi SH, Lim CM, Koh Y, et al: Comparable efficacy of tigecycline versus colistin therapy for multidrug-resistant and extensively drug-resistant Acinetobacter baumannii pneumonia in critically ill patients. PLoS One 2016; 11: e0150642.

27) Durante-Mangoni E, Signoriello G, Andini R, Mattei A, De Cristoforo M, Murino P, et al: Colistin and rifampicin compared with colistin alone for the treatment of serious infections due to extensively drugresistant Acinetobacter baumannii: a multicenter, randomized clinical trial. Clin Infect Dis 2013; 57: $349-58$
28) Abdellatif S, Trifi A, Daly F, Mahjoub K, Nasri R, Ben Lakhal S: Efficacy and toxicity of aerosolized colistin in ventilator-associated pneumonia: a prospective, randomized trial. Ann Intensive Care 2016; 6: 26 .

29) Garnacho-Montero J, Ortiz-Leyba C, Jiménez-Jiménez FJ, Barrero-Almodóvar AE, García-Garmendia JL, Bernabeu-Wittell M, et al: Treatment of multidrug-resistant Acinetobacter baumannii ventilatorassociated pneumonia (VAP) with intravenous colistin: a comparison with imipenem-susceptible VAP. Clin Infect Dis 2003; 36: 1111-8.

30) Esperatti M, Ferrer M, Giunta V, Ranzani OT, Saucedo LM, Li Bassi G, et al: Validation of predictors of adverse outcomes in hospital-acquired pneumonia in the ICU. Crit Care Med 2013; 41: 2151-61.

31) Chan JD, Graves JA, Dellit TH: Antimicrobial treatment and clinical outcomes of carbapenem-resistant Acinetobacter baumannii ventilator-associated pneumonia. J Intensive Care Med 2010; 25: 343-8. 